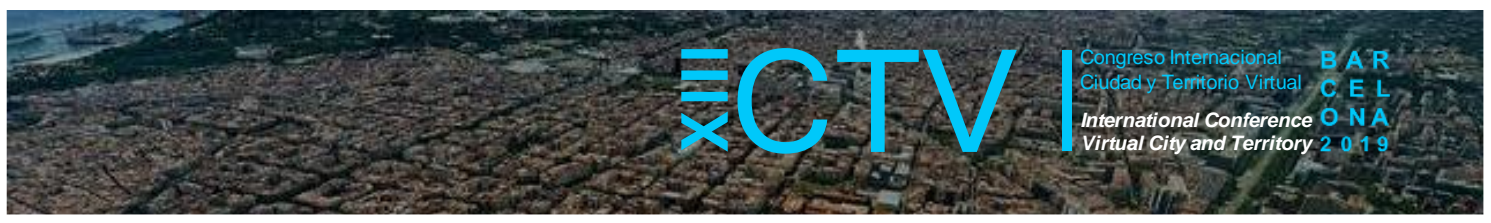

\title{
ACERCAMIENTO DOCUMENTAL A LOS CONCEPTOS ENERGÉTICOS EN LA CIUDAD TRADICIONAL
}

\author{
Coll-Pla, Sergio ${ }^{1 *}$; Costa, Agustí ${ }^{2}$; Mallafrè, Cèlia ${ }^{3}$; Modinos, Ferran ${ }^{4}$; Bas, Melanie ${ }^{5}$; Moreno, \\ David ${ }^{6}$, Puche, Josep Maria ${ }^{7}$ y Sardà, Jordi ${ }^{8}$
}

Remisión inicial: 2019-06-12; Remisión definitiva: 2019-10-16; Publicación: 2019-12-21

Citación: Coll-Pla, S. et al. (2019). Acercamiento documental a los conceptos energéticos en la ciudad tradicional. En XIII CTV 2019 Proceedings: XIII International Conference on Virtual City and Territory: "Challenges and paradigms of the contemporary city": UPC, Barcelona, October 2-4, 2019. Barcelona: CPSV, 2019, p. 8535. E-ISSN 2604-6512. DOI http://dx.doi.org/10.5821/ctv.8535

Resumen

En el presente contexto de internacionalización de la economía, el replanteamiento de los sistemas urbanos y de competitividad entre metrópolis, vemos en el pasado la oportunidad de buscar algunos ejemplos que nos ayuden a mejorar las propuestas de presente-futuro. El urbanismo y la arquitectura contemporánea no pueden ser comprendidos sin incluir en su definición un replanteamiento hacia lo sostenible, tratando de dar respuesta a las numerosas preocupaciones climáticas y sostenibles que desafían la habitabilidad de las ciudades. En este sentido, las propuestas urbanas que buscan propiciar un contexto más acorde con las lógicas medioambientales actuales parecen compartir, al menos, una problemática: unos sistemas urbanos considerados obsoletos, ciudades que en muchas ocasiones no son comprendidas, o no son leídas conforme las importantes posibilidades que presentan.

\begin{abstract}
In the present context of the economy's internationalization, reconsidering the urban systems and the competitive between metropolis, we see in the past the opportunity to find some examples which can help us to improve the projects in present-future. The urbanism and the contemporary architecture cannot be understood without including in its definition a rethinking towards something sustainable, trying to answer large climatic and sustainable worries, which challenge the habitability in the cities. In this sense, the urban proposal which are looking for propitiate a context more consistent with the actual environmental logics seem to share, at least, one problematic: urban systems are considered obsoletes, cities which in many occasions are not understood, or are not read according the important possibilities presented.
\end{abstract}

Palabras Clave: Ciudad antigua; ciudad medieval; sostenibilidad; energía

Key words: Ancient city; medieval city; sustainability; energy

\section{Introducción y objetivo}

Las ciudades actuales son fruto de planes de crecimiento donde la expansión se planteaba como infinita. Este crecimiento se plantea como parte de un sistema de agregación que facilita la repetición de módulos hasta la saciedad, como es el caso del ensanche, en el que su inicio y final está condicionado por la retícula. Si se realiza un estudio del urbanismo a lo largo de la historia observamos que existen diferentes tipos de agregaciones, todos ellos condicionados por principios mayoritariamente comerciales o sociales.

\footnotetext{
1 Profesor del Doctorado Industrial, Escuela de Arquitectura de Reus, Universitat Rovira i Virgili, URV, https://orcid.org/0000-0002-4718-5810; ${ }^{2}$ Profesor de la Escuela de Arquitectura de Reus, URV, https://orcid.org/00000002-6194-3243; ${ }^{3}$ Profesor de la Escuela de Arquitectura de Reus, URV, https://orcid.org/0000-0003-4332-0090; ${ }^{4}$ Profesora asociada de la Escuela de Arquitectura de Reus; URV ${ }^{5}$ Escuela de Arquitectura de Reus, URV; ${ }^{6}$ Escuela de Arquitectura de Reus, URV; ${ }^{7}$ Instituto Catalán de Arqueología Clásica, ICAC, ${ }^{8}$ Escuela de Arquitectura de Reus, URV. *Correo de contacto: sergio.coll@urv.cat
} 


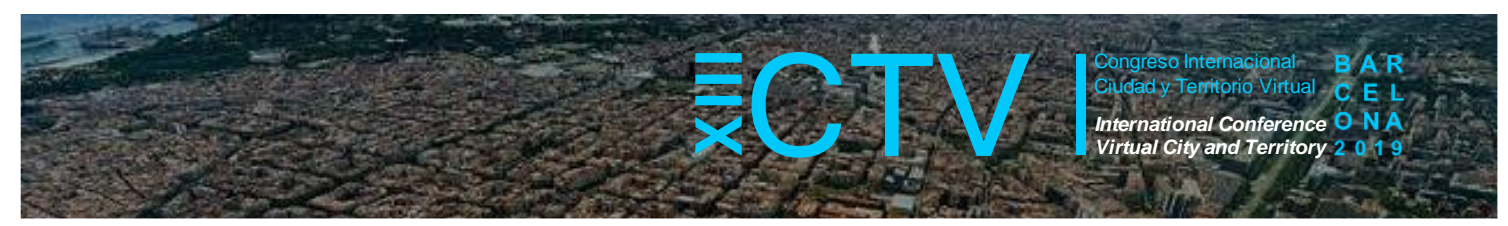

En la actualidad, se pueden diferenciar dos tipos de urbanismo: el proveniente de la ciudad antigua, que es la ciudad pública; y el originario en la industrialización, que es la ciudad doméstica (Oliva, 2013). Este estudio se centrará en la comprensión de la ciudad pública ya que ésta recurre a estrategias pasivas para su correcta habitabilidad (Ros, 2017). Será en el urbanismo romano donde se encuentran más indicios de que éste es fruto de una reflexión global, principalmente reflejando el macrocosmos y microcosmos. Los edificios romanos nacen de una estrecha relación con el entorno natural, basada en el estudio de características geométricas, variables climáticas (presión, temperatura y humedad relativa, estado del cielo, régimen del viento, precipitaciones, luminosidad...), parámetros geográficos (latitud, relación entre masa de aguas subterráneas y superficiales...) y topográficos (altura, orientación, estructura del suelo, dirección de vientos predominantes). (Lucchi, 2015).

En épocas posteriores, como la Edad Media se observa un cambio de interés pasando a ser un urbanismo organicista, resultado de las necesidades comerciales. El urbanismo Renacentista, en cambio, parte de la búsqueda de la centralidad, siendo utópico, y se caracteriza por la realineación de calles. Es evidente que cada uno de estos tipos de urbanismo permitió la continuidad de la sociedad que todavía las habita, llegando a las dudas actuales sobre la pervivencia de la humanidad en la actualidad y en el futuro.

Las previsiones más catastróficas prevén una ciudad con una dependencia absoluta de los recursos energéticos activos, siendo estos tremendamente perjudiciales para la reducción de las tendencias pesimistas. Estas tendencias en muchas ocasiones están apoyadas por estudios científicos que afirman que la humanidad nunca ha sufrido el futuro que nos espera. Aceptando este hecho debemos asumir también que la sociedad actual vive con unos estándares de confort tremendamente altos, hecho que tampoco se ha repetido en la historia.

Por ello, el objetivo de esta publicación es el de realizar una aproximación teórica hacia las técnicas constructivas y estudios vinculados a los sistemas urbanos del pasado, con el fin de buscar en ellos posibles respuestas a preguntas del presente, y de esta manera reaprender lo que el paso del tiempo ha provocado el olvido de estas civilizaciones. Haciendo una comparación crítica entre las ciudades de hoy y del ayer, se podrán extraer los beneficios de las épocas anteriores para intentar solucionar problemas actuales.

Como primera fase de la investigación se ha partido de una búsqueda bibliográfica para generar una base documental que permita contextualizar y avanzar en la investigación. A partir de esta búsqueda se puede realizar un seguimiento histórico a nivel energético suficientemente amplio y documentado. También se observa como en cada época y/o lugar se desarrollan técnicas en ámbitos de estudio distintos buscando diferentes soluciones a una misma pregunta.

Una segunda fase de trabajo incidirá en la búsqueda de parámetros a estudiar que nos permitan parametrizar energéticamente la ciudad. El estudio de las energías en los edificios se limita al estudio del confort acústico, confort lumínico y térmico, teniendo en cuenta las condiciones higrotérmicas (Temperatura ambiental, Temperatura radiante, Humedad Relativa, Velocidad viento).

La investigación finalizará en la aplicación de estos datos en un ejemplo de estudio adecuado que nos permita la comparación energética a lo largo de la historia. La generación de los datos que luego serán usados se hará a partir de un soporte gráfico. El redibujo a través del software 


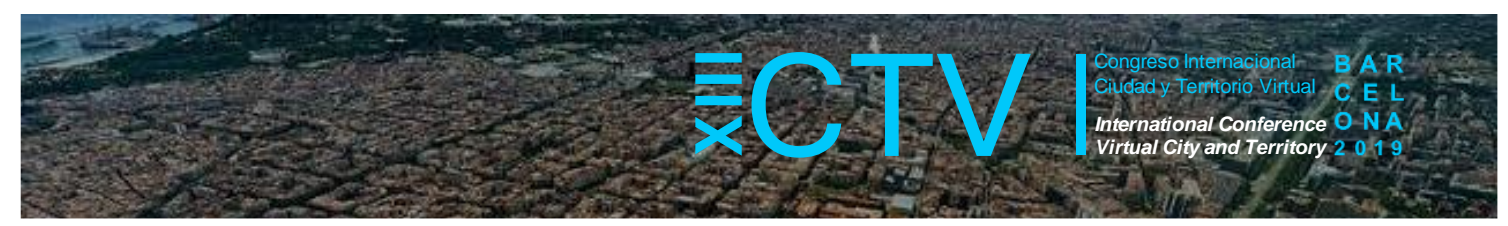

Autodesk AutoCAD (versión 2018), herramienta de dibujo altamente extendida en el mundo de la arquitectura y la ingeniería, que proporcionará unas planimetrías con las cuales podremos tomar datos de áreas y volúmenes, proporciones, y comparación de vacíos y llenos de manera automática y precisa. La superposición entre las dos planimetrías puede dar una clara imagen de los cambios de tipología de urbanismo de una manera directa.

\section{Búsqueda bibliográfica sobre aspectos energéticos en las ciudades pasadas}

Los focos para el estudio son la ciudad Romana, la ciudad Medieval y la ciudad Renacentista, pues son representativas de la evolución de la ciudad a lo largo de tres épocas históricas distintas.

\subsection{La ciudad romana, el primer foco de estudio}

En el Imperio Romano se crearon entornos monumentales en los que el espacio; la luz y la sombra; la ventilación; etc. sintonizaron entre sí. Las termas y los sistemas de calefacción como el hipocausto fueron el inicio del desarrollo de sistemas activos de climatización.

La ciudad romana se ordenaba a partir de dos ejes ortogonales, conocidos como el cardus y el decomanus, que ordenaban el microcosmos, confiriéndole un carácter de totalidad a la ciudad y sus calles. El cardus, orientado de norte a sur, es uno de los ejemplos de elección de la orientación según el cosmos, donde el centro o mundus era el encargado de relacionarse con las fuerzas terrestres. (Norberg, 2007)

Los romanos no entendían el concepto de sostenibilidad desde el punto de vista de la climatización, pero se creían parte de un todo que debían respetar, por lo que sus ciudades garantizaban el aprovechamiento de la ventilación, el asoleo y la humedad relativa.

Desde el punto de vista de la climatización deben nombrarse los edificios dedicados a las termas, los cuales seguían la orientación de la ciudad y estas mantenían un orden microcósmico semejante al de la ciudad. (Norberg, 2007). En ninguna situación se deduce que, la organización de éstas responde a una lógica climática, sino más bien obedece a una lógica espiritual y simbólica. Cierto es que se acepta que una vez marcado el cardo y decomano, se permitía cierta adaptación a la topografía o las preexistencias (Roth, 2003). A la práctica, las termas eran edificios complejos que requerían de un aporte energético con el objetivo de garantizar unas condiciones de confort (Temperatura y humedad altas), pero que de ser estudiados se deben buscar similitudes con edificios completamente aislados.

Respecto al confort térmico y lumínico la tradición romana aporta los escritos de Sócrates (470 - 399 a.C., citado por Jenofonte) en los que se explica cómo la orientación sud incide más el sol en invierno que en veranoMás concreto será Marco Vitruvio Polión (80 - 15 a.C. aprox.) donde en los Diez Libros de Arquitectura acepta que las edificaciones deben adaptarse según la longitud y la latitud, además de adaptarse al clima imperante en la zona, diferenciando las construcciones de los climas fríos respecto de los cálidos. El mismo Vitruvio habla sobre efectos sonoros, pero no lo relaciona con el confort, pero cierto es que la vivienda romana de por sí ya se aislaba de la calle. (Vitruvio, 2000). 


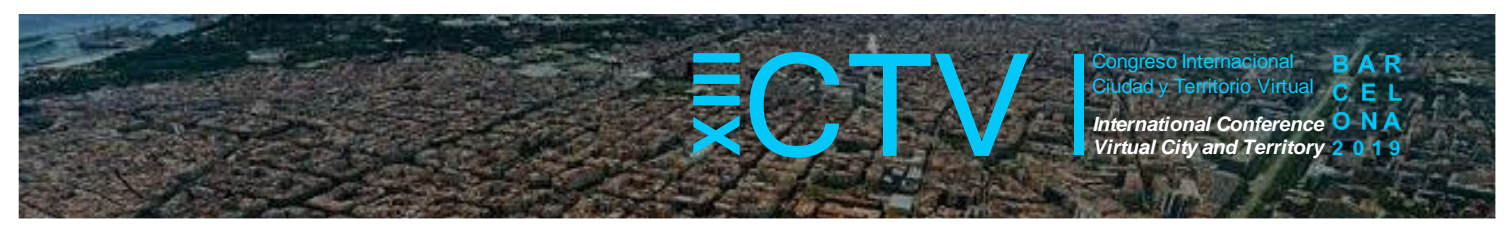

Otro factor a tener en cuenta es la tipología de vivienda en la ciudad romana, conocida como Domus. La casa romana se articulaba a partir de un patio, incluyendo algunas veces un impluvium. Esta tipología y su relación natural con los factores climáticos tales como temperatura, humedad y ventilación favorecía unos parámetros aptos para la habitabilidad de la vivienda

\subsection{La ciudad medieval}

La Edad Media estuvo fuertemente marcada por las distintas religiones. Mientras los cristianos utilizaban la luz celestial como símbolo para generar grandes efectos teatrales, en el mundo islámico recurrían a la sombra y la ventilación para emular las sensaciones de un paraíso en la Tierra.

La ciudad de la Edad Media tiene formas diferentes, sí que existe una derivación de la ciudad romana, pero se le tiene que sumar diversos esquemas que responden a las necesidades comerciales y sociales. En ningún caso se tiene constancia de que estos esquemas respondan a algún principio energético para su desarrollo. Existe la ciudad lineal, la crucial y la nuclear. En todas ellas se le debe sumar un fuerte componente organicista (Franchetti, 1985).

La ciudad islámica se plantea como herencia de la metrópolis romana, planteando su urbanismo a partir de un tablero isodómico. A diferencia de la romana, la islámica no necesariamente tiene una orientación norte - sur. (Acién, 2003). También existen casos donde la ciudad puede ser orgánica, espontánea o irregular (Navarro; Jiménez, 2007). Aun así, como se puede ver, todos los casos responden a una lógica comercial y equitativa. En el caso de los edificios, cada región o clima requerían de un determinado uso de la luz, sombra y ventilación, adaptándose así a las necesidades de sus habitantes.

\subsection{La ciudad renacentista}

El Renacimiento recuperó los espacios de transición (logia y el atrio) como piezas de unión entre arquitectura y naturaleza, ensanchó y alineó calles, todo ello siempre buscando unas proporciones, una axialidad y un paisaje. El urbanismo renacentista toma una voluntad mucho más ordenada, geométrica y homogénea, que recuerda a la época Romana. Se genera un nuevo interés por las perspectivas y las proporciones, con una visión muy diferente de la ciudad de principios de la Edad Media. Leon Baptista Alberti en De re aedificatoria o Leonardo Da Vinci, entre otros, recuperaron la figura de Vitruvio, pero se centraron en conceptos estéticos no funcionales (Páez, 2010). Su ciudad ideal era una unión entre un lugar concreto y homogéneo junto a la centralización, "entre todas las ciudades, la mejor es la que tiene planta circular" Leon Baptista Alberti. La citación de Alberti muestra la idealización de las formas puras que había en el renacimiento. En esta época se creía que la forma pura era la circular, de ahí que se pretendiera construir ciudades radiales, de tal forma que en el centro del círculo se colocara la institución más importante. De esta manera, las calles se conformaban mediante desfases de este círculo central, acción que influya en parámetros cómo el viento. (Norberg, 2007) Durante esta época el urbanismo se limitó en la expansión de les áreas urbanas o la remodelación parcial de las ciudades. Los principales elementos de estos planteamientos urbanísticos fueron, la creación de calles principales que facilitaban la movilidad de la ciudad y la retícula como estrategia para formar nuevos barrios que serían la base para una futura expansión de la ciudad. 


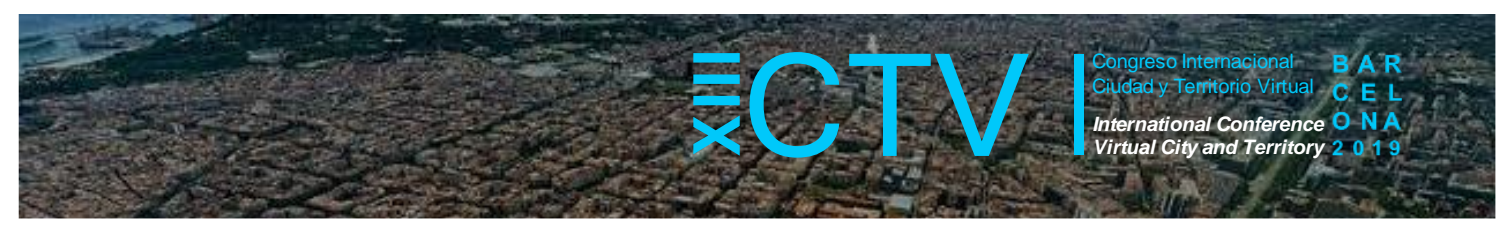

Otro punto importante es la época renacentista son las ciudades coloniales. Las nuevas ciudades coloniales recuperaron la centralidad y el orden, con lo que ello supuso un nuevo acercamiento a la naturaleza, suponiendo una vuelta a la ciudad romana, hecho que se observa por ejemplo en la ciudad de Arequipa, donde se desarrolla una retícula casi cuadrada que, sin seguir los ejes este-oeste, sí que se desarrollan unas edificaciones de baja altura que permiten el asoleo de las calles. También las "Casonas", que desarrollan su vida alrededor de patios interiores y traseros, con lo que alejan la vida privada de las calles. Todo ello la acerca a la ciudad romana.

\section{Parámetros a estudiar de la ciudad}

Para poder tener un estudio completo de la ciudad es obligatorio realizar un análisis histórico y de sus funciones, una auditoria energética, las posibles variaciones del clima, una evaluación de la compatibilidad y un estudio de la reversibilidad de cada acción (Lucchi, 2011). Hay estudios urbanos actuales que se basan en la medición de la temperatura del aire, la temperatura de superficie, la humedad relativa y la velocidad del aire (Johansson, 2006).

Cabe destacar que se puede encontrar un estudio que no se basa en tomar datos climáticos directos, sino en aspectos relacionados en la composición de la ciudad. Estos son la densidad de edificación, la compacidad de los edificios, los elementos impermeables (pavimentos) y el porcentaje de viales También, se pueden tener en consideración detalles arquitectónicos, como los balcones, que pueden tener un fuerte impacto en el análisis general del confort del viento en los diferentes espacios. (Johansson, 2006).

No hay estudios al respecto, pero los sistemas de clasificación y certificación de edificios sostenibles establecen diferentes categorías de estándares que evalúan la presencia o la ausencia de determinados factores relacionados con la calidad ambiental en las diferentes etapas de la edificación (diseño, construcción, uso, reutilización, demolición).

En la actualidad los estándares de construcción, establecidos por los sistemas de certificación, surgen de la normativa local e internacional. Cada uno de los sistemas de clasificación y certificación representa un enfoque o filosofía propia, según el lugar donde se ha concebido, pero con un objetivo en común: hacer que los edificios sean cada vez más sostenibles, por ende, un mejoramiento continuo. Estos sistemas, brindan unas pautas estándares sobre las opciones de diseño y/o construcción que son ecológicamente responsables, a la vez que entregan premios y reconocimientos públicos al esfuerzo (Cicely, 2009).

En referencia a los estudios que se llevan a cabo en la actualidad sobre la ciudad y/o zona urbana, mayoritariamente se hace uso de dispositivos electrónicos para medir los parámetros de temperatura, humedad, porcentaje de $\mathrm{CO}_{2}$, etc. Por lo que hay que buscar otros parámetros a analizar gracias a estudios arqueológicos y arquitectónicos que nos dan a conocer cómo en civilizaciones pasadas componían y construían los centros urbanos. Estos parámetros a estudiar son la tipología de urbanismo, la densidad de construcción y la compacidad de los edificios; parámetros que, gracias a los estudios nombrados anteriormente, se pueden cuantificar y comparar.

Es por ello, que a partir de todas las referencias mencionadas anteriormente hemos visto conveniente hacer un análisis de los parámetros, tales como, la composición de la ciudad, la 


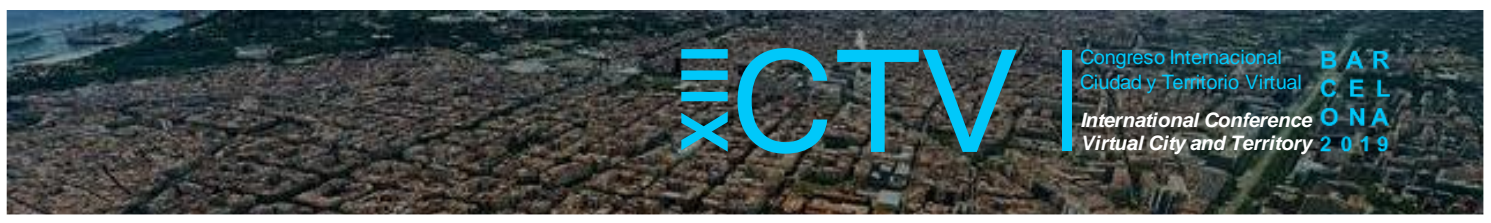

densidad de la edificación, los detalles arquitectónicos, etc. Tomando como ejemplo la ciudad de Barcelona.

\section{Ejemplo de estudio: La ciudad de Barcelona}

La ciudad de Barcelona primero fue conocida como Barcino, una pequeña ciudad de fundación romana que con el paso de los siglos se convirtió en una importante capital medieval. Barcino, una ciudad con la tipología estándar romana, con cardus y decomanus bien marcados y definidos que formaban una planta reticulada, es un ejemplo muy claro de transformación que sufre en la edad del medievo con el centro urbano conocido como "barrio gótico", un centro lleno de irregularidades, espacios estrechos y con formas orgánicas. Hay unas suposiciones de la ciudad romana consensuada que nos servirá como base de estudio de la época romana y, el barrio gótico, el cual se conserva en un estado casi invariable des de su origen. Gracias a estas dos fuentes documentales se podrán hacer las dos comparativas de los parámetros a estudiar.

Cuando llegaron los romanos, entre los años 15 y 13 a. C., buscaron el mejor sitio para fundar una colonia situada entre las prósperas Emporiae (Empúries) en la costa norte, y Tarraco (Tarragona) en la costa sur de Cataluña. El lugar escogido fue la cima del Mons Taber, un pequeño cerro frente al mar que dominaba la llanura. La colonia, con el nombre completo de Iulia Augusta Faventia Paterna Barcino, creció rápidamente, y entre los siglos I y II se amuralló. Posteriormente se reforzaron las murallas en los siglos III y IV y se delimitó un perímetro que se mantendría hasta la Edad Media. También se mantuvo la estructura propia de una ciudad romana, de retícula definida por los ejes cardus y decomanus, que ordenaban todo el recinto. La existencia de grandes domus que se repartían dentro de la ciudad, con jardines y ricas decoraciones ornamentales, confirma la presencia de grandes familias propietarias. Y, gracias a estas familias, la pequeña Barcino fue agrandándose y adquiriendo relevancia.

Al configurarse en el siglo XII el Principado de Cataluña, y bajo la protección de los condes, la Barcelona agrícola y artesanal ganó relevancia política y comercial, lo que causó un gran crecimiento de la ciudad. Las antiguas murallas romanas se quedaron pequeñas, y a las afueras, en torno a nuevas parroquias, se crearon densos núcleos urbanos que quedaban desprotegidos.

Con la expansión política y comercial se produjo un tiempo de esplendor que propició un crecimiento urbanístico. Primero, en el siglo XIII, se construyó una nueva muralla que amplió la ciudad. Después, en el siglo XIV, se iniciaron las obras para ampliar aún más este recinto amurallado, que incluyó el barrio del Raval, extendiéndose hasta alcanzar el actual Paral-lel y cerrándose por mar. Durante esta expansión en época medieval, poco a poco se fueron desdibujando los trazados romanos, llegando a perder casi la totalidad de la ordenación urbana previa. Esto dio paso a un nuevo urbanismo medieval, mucho más compacto, organizado a partir de la Plaza Sant Jaume, situada en el centro del núcleo. Con el paso de los siglos, la ciudad fue creciendo y ampliándose hacia el exterior, pero aun así conservó gran parte de la estructura urbana medieval del núcleo de la ciudad, denominándolo y conociéndolo actualmente como Barri Gòtic.

Es por ello que para realizar el estudio se escoge como ejemplo esta ciudad, por el hecho de tener una gran cantidad de información sobre su creación y evolución hasta nuestros días. Su origen romano documentado y su posterior ampliación en la Edad Media son un claro ejemplo 


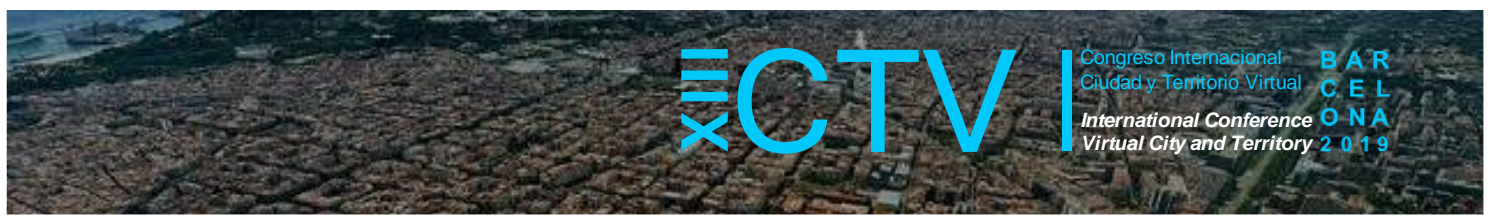

de la evolución del urbanismo de las ciudades del clima mediterráneo. A partir de la información gráfica existente sobre estos periodos se desarrollará la comparación de ciertos parámetros urbanos, tales como densidad edificada, espacios urbanos, y volumen construido con tal de llegar a una posible comparación de la ciudad entre estas dos épocas.

\section{Resultados}

El principal resultado que se obtiene es la caracterización de diversos modelos urbanos, así como los datos analizados en la ciudad de Barcelona. A lo largo de la historia, la arquitectura popular ha sabido adaptarse a las condiciones del entorno en busca del máximo aprovechamiento energético apoyándose en la experiencia y el sentido común.

Un estudio historiográfico indica que durante la época Renacentista la ciudad ya había crecido por fuera del límite generado por las murallas romanas, expandiendo la ciudad hacia sus alrededores. Aun así, mantenía el centro en el mismo lugar donde había el núcleo antiguo de la ciudad Romana. Su crecimiento trajo un aumento de la muralla mucho mayor a la Romana, y creando unas nuevas áreas urbanas mucho mayores, y una calle principal rectilínea muy característica del Renacimiento. Si nos enfocamos en el sitio analizado durante la época romana y medieval, nos damos cuenta que no se ha producido un gran cambio, ya que la gran diferencia fue el crecimiento hacia las afueras de las murallas. De esta manera creemos que el gran cambio que se produce en nuestro emplazamiento es durante el cambio de la época Romana a la Medieval.

Tabla 1. Tabla de resultados obtenidos sobre la comparación entre épocas del Barri Gòtic de Barcelona

\begin{tabular}{l|c|c}
\hline & ÉPOCA ROMANA & ÉPOCA MEDIEVAL \\
\hline Espacio construido $\mathbf{~ m}^{2}$ & 28.689 & 37.128 \\
\hline Espacio público $\mathbf{m}^{2}$ & 17.825 & 18.502 \\
\hline Espacio vacío $\mathbf{m}^{2}$ & 5.087 & 630 \\
\hline Área total $\mathbf{m}^{2}$ & 51.601 & 56.260 \\
\hline Vacío/Construido & $15,06 \%$ & $1,67 \%$ \\
\hline Espacio público/Construido & $34,54 \%$ & $32,89 \%$ \\
\hline Volumen construido $\mathbf{m}^{3}$ & 143.445 & 334.152 \\
\hline
\end{tabular}

Los resultados muestran que durante la época medieval la ciudad sufrió un pequeño crecimiento, que queda reflejado en el área total de las dos épocas. Este crecimiento se vio condicionado a la muralla que protegía la ciudad. Pero por otra parte la ciudad se densifica, el espacio construido aumenta mucho más, y si comparamos el crecimiento total con el del espacio construido, es casi el doble. La ciudad aumenta $4.659 \mathrm{~m}^{2}$ y la construcción $8.439 \mathrm{~m}^{2}$.

Esta diferencia es más clara al fijarnos en el volumen construido, donde vemos que la diferencia aumenta a casi $200.000 \mathrm{~m}^{3}$ entre épocas. Para conseguir estos datos hemos considerado que en la época romana la edificación más común era de $\mathrm{Pb}+1$, de unos 5 metros y en la época medieval aumenta a edificaciones de $\mathrm{Pb}+2$ consiguiendo una altura de unos 9 metros. El espacio construido aumenta y el espacio vacío disminuye.

Viendo los porcentajes de los datos podemos decir que el espacio público respecto al construido no tuvo una gran variación respecto a las épocas, teniendo las dos épocas unos 


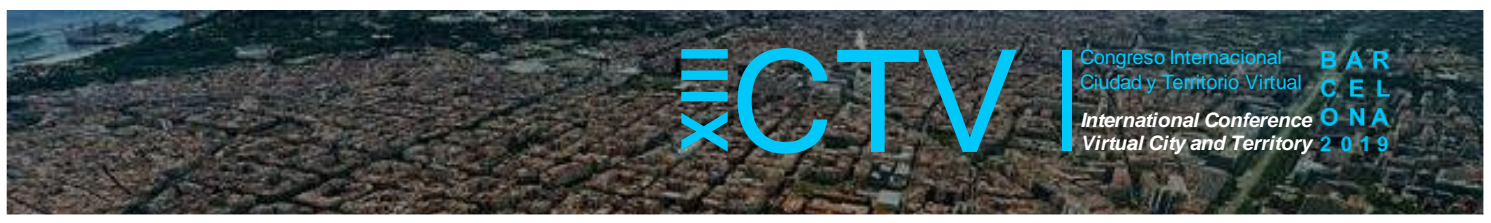

valores muy parecidos (E.R 34,54\%, E.M 32,89\%). Donde sí se nota una variación de porcentaje es en la relación de espacio vacío con el construido, bajando en la época medieval a una cifra muy menor a la romana (E.R 15,06\%, E.M 1,67\%), esta disminución es debida al cambio de tipología que sufrieron las viviendas.

Figura 2. Comparación gráfica realizada sobre el urbanismo romano y el medieval
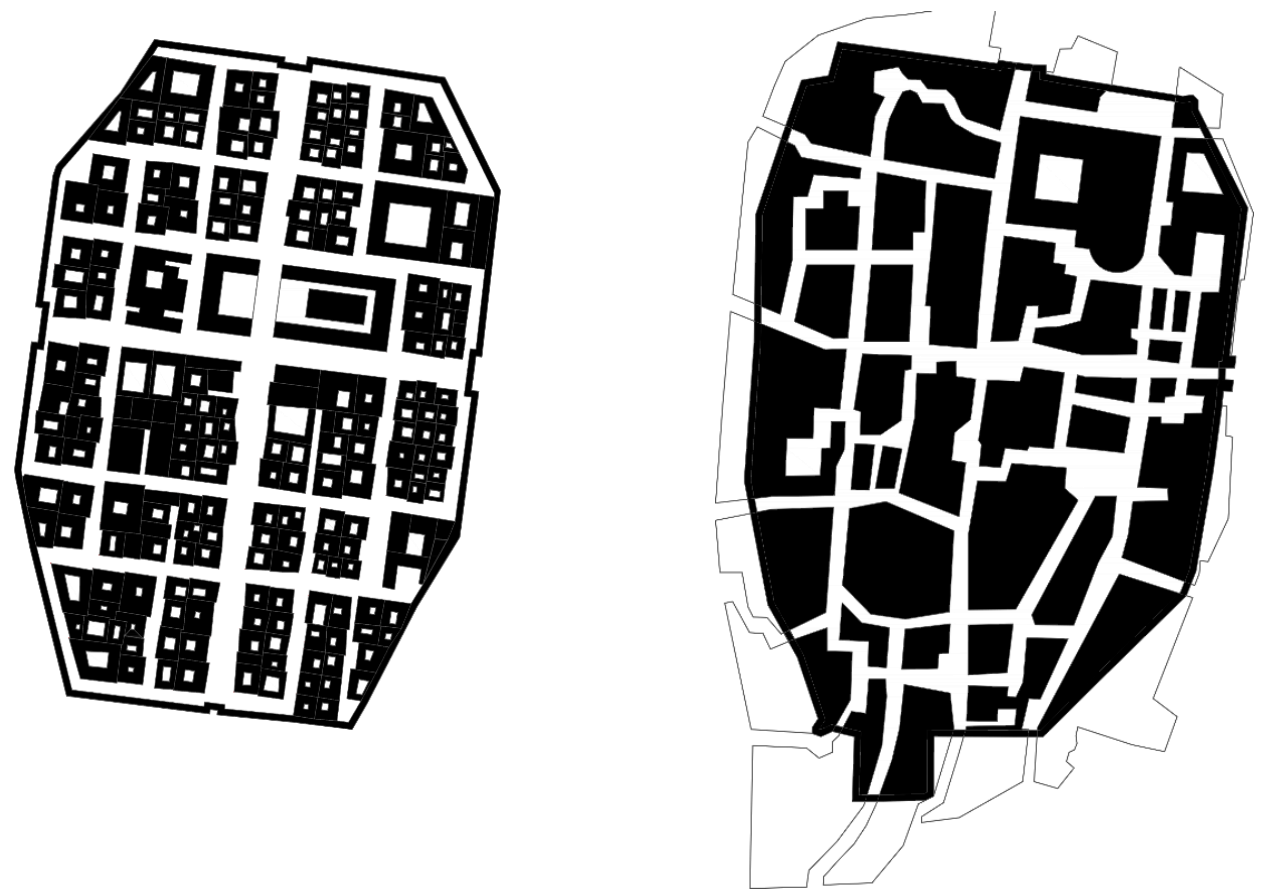

Nota: Se puede observar las diferentes densidades en cada época y cómo en la medieval la ciudad se densifica.

\section{Discusión}

Una vez analizados los resultados observamos que un aumento de altura en la ciudad medieval genera unas condiciones climáticas diferentes a las Romanas, pudiendo variar en el viento, las temperaturas y la variación que habrá entre las mínimas y las máximas, dado a una mayor incidencia del sol y un cambio en la cantidad y dirección del viento que habrá en las calles.

La disminución del vacío en la ciudad provocó una reducción del confort térmico. Diversos estudios demuestran la importancia de los espacios vacíos entorno a las temperaturas de la ciudad. Otro factor muy importante es la vegetación, en este análisis nos basamos en la idea de la existencia de patio en las casas particulares. Estos patios tienen un papel muy importante para entender cómo eran las condiciones en la ciudad Romana. El uso de vegetación en estos espacios mejoraba las condiciones climáticas de la ciudad, de manera que la percepción del espacio se veía afectado de manera favorable gracias al confort térmico.

En la actualidad una ciudad sin calidad climática genera un confort no óptimo y a la vez se necesita más energía. Vemos que en la ciudad Medieval hay una densificación de la ciudad con la que conlleva una calidad climática peor. Este incremento de densidad provoca un 


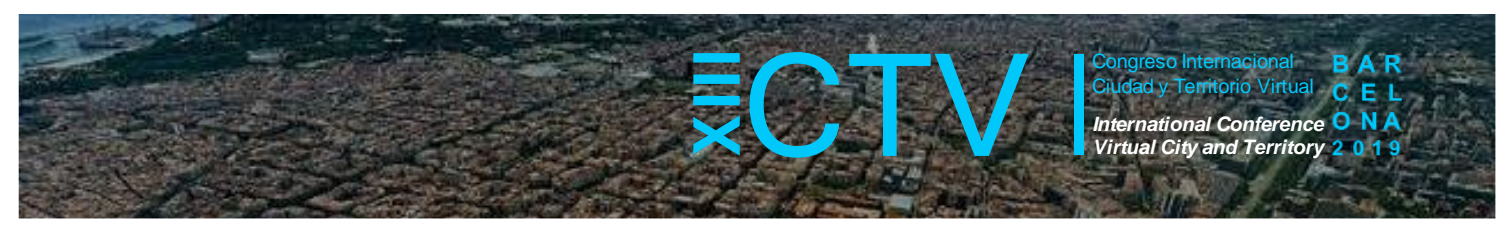

aumento de la temperatura y de la humedad por lo que el clima es más frio. También se produce un desarrollo mayor de la inercia térmica, atemperando los climas.

Si también se tiene en consideración que en la ciudad medieval la tipología de casa varia con respecto a la romana, perdiendo el patio como elemento propio de la vivienda, se produce un cambio en las condiciones de confort. Esto no solo ocurría en la vivienda sino también en las calles, ya que el hecho de poseer en una ciudad una gran cantidad de casas con patio influía sobre el urbanismo, y por lo tanto sobre el clima de la ciudad. Es por ello que podemos afirmar que los habitantes de la época medieval no pudieron llegar a tener una sensación de confort dentro de su localidad.

\section{Conclusiones}

Gracias al estudio realizado en estas dos épocas podemos ver que el suceso más importante que ocurre es la densificación de la ciudad, junto con un aumento de la población.

El crecimiento de la ciudad queda limitado por la muralla ya existente en la época romana. De manera que la ciudad no aumento casi nada en extensión, pero se densifico más en su centro y creció en altura. Este cambio se produjo variando las tipologías de casas ya existentes, pasando de una vivienda de planta baja o planta baja más uno, normalmente con patio, a unas tipologías de casas más altas con planta baja más dos. Esta variación conllevó a una reducción del espacio vacío dentro de la construcción, alterando así los parámetros climáticos de la ciudad.

En ciudades con densidades altas el consumo de energía es más alto en los interiores, además de tener un mayor impacto en el ambiente. Pueden provocar turbulencias en el viento y descenso de la velocidad de este generando así una disminución de refrigeración, lo cual es negativo en épocas de calor. También los edificios altos producen sombras solares y pluviométricas, en ciudades medievales puede ser una desventaja si tenemos en cuenta la distancia cercana de los edificios, ya que la incidencia solar seria mínima, hecho que empeora la calidad ambiental.

En cambio, la reducción del tamaño o una densidad baja mejora el microclima de la ciudad, por ejemplo, en lo que se refiere a temperatura suelen ser más bajas que en densidades altas ya que en estas se produce el efecto "isla de calor" aumentando la temperatura y provocando así un ambiente no deseado. Esto se debe por ejemplo al pavimento como la piedra y el ladrillo, además de otros materiales que poseen una gran inercia térmica y que, por ende, almacenan el calor aumentando así la temperatura.

Un estudio, analizó y llegó a la conclusión de que se podría llegar a las mismas condiciones favorables de la densidad baja en una ciudad con densidad alta cambiando de forma estratégica los detalles arquitectónicos. De tal forma que no fueran un estorbo para la circulación del viento, la radiación solar y la calidad del aire (Ruano, 2012).

Para mejorar la calidad de las ciudades del futuro podríamos utilizar algunas estrategias de la arquitectura popular, como puede ser: la flexibilidad ante la radiación solar (captación / protección), la flexibilidad en el diseño de los cerramientos (masa térmica/aislamiento térmico), el enfriamiento evaporativo y radiante, y el aprovechamiento de la ventilación. 


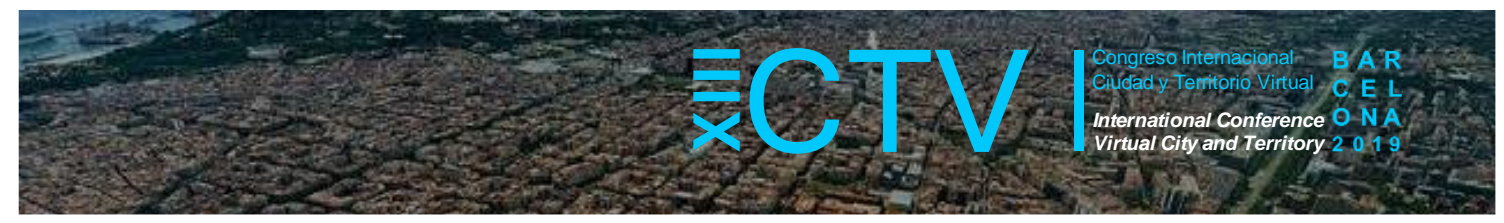

También se debe intentar estabilizar la temperatura interior para llegar al confort. Por ello, según las diferencias climáticas de la región se deben aplicar estrategias diversas. Algunas de estas estrategias en la zona con clima mediterráneo son espacios públicos soleados (pero con soportales para protegerse del sol en verano y de la lluvia), la presencia de patios autosombreados por el edificio donde se pueda producir el enfriamiento radiante o evaporativo, voladizos que protejan del sol y/o de la lluvia las fachadas, muros gruesos y pesados para dotar al edificio de mucha masa térmica, incorporación de materiales aislantes térmicos ( paja, madera, cámaras de aire, piedras porosas, etc.), edificios enterrados o semienterrados para incrementar el efecto de masa y del aislante térmico, y el aprovechamiento de la ventilación cruzada (Neila, 2004).

Las investigaciones en el futuro deberán ahondar en las energías intrínsecas de la ciudad, entre varias se deberá tantear las posibilidades de la exergía. La exergía determina cuantitativamente el valor termodinámico de cualquier recurso, estableciendo pautas para su ahorro y uso eficiente. Es evidente que las ciudades contienen una temperatura más alta respecto a su entorno próximo, con lo que se debe considerar esto como posible energía a aprovechar.

Contribuciones de los autores: Todos los autores han contribuido de igual manera en el desarrollo del artículo.

Conflicto de Intereses: Los autores declaran que no hay conflicto de intereses.

\section{Bibliografía}

Acién Almanda, M. (2003). La formación del tejido urbano en Al-Andalus. Universidad de Málaga. Màlaga, Espanya.

Cicely Enright (2009). LEED, Green Globes y ASTM. Clasificación de edificios verdes.

Franchetti Pardo, V. (1985). Historia del Urbanismo, Siglos XIV y XV. Instituto de Estudios de Administración Local.

Johansson, E. (2006) Influence of urban geometry on outdoor thermal comfort in a hot dry climate: A study in Fez, Morocco. Building and Environement. 1326-1338

Lucchi, E. (2015). Sostenibilidad y eficiencia energética de los edificios histórico.

Lucchi, E. (2011). Efficiency in Historic Building. A tool for Analysing the compatibility, integration and reversibility of renewable energy technologies. World Renewable Energy Congress. Suecia, Low-Energy Architecture (LEA).

Navarro Palazón, J.; Jiménez Castillo, P. (2007). Algunas reflexiones sobre el urbanismo.

Neila González, F. J. (2004). Arquitectura bioclimática en un entorno sostenible. Ed. Munilla Lería. 


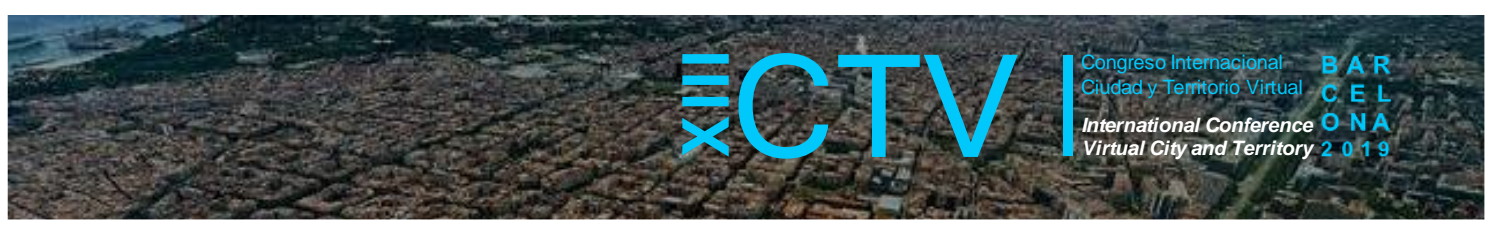

Norberg - Schulz, C. (2007). GG Reprints. Arquitectura occidental. Ed. Gustavo Gili, SL. 1aㅗ Edición, 6a tirada. En Capítulo III: La arquitectura romana. Introducción, paisaje y asentamiento. Pp. $44-46$.

Oliva Casas, J. (2013). Models de ciutat i ecologisme. Pp. $105-112$.

Páez García, A. (2010). Sostenibilidad urbana y transición energética: un desafío institucional.

Roth M. L. (2003). Entender la arquitectura sus elementos historia y significado. Ed. Gustavo Gili, SL. 1를 Edición, 3atirada. Capítulo 12: Arquitectura romana. Urbanismo romano. Pp. 231.

Ros García, J. O. (2017). Arquitectura Biosaludable: parámetros de habitabilidad. Pp. 92 - 95

Ruano, M. (2012). Un Vitruvio ecológico. Principios y práctica del proyecto arquitectónico sostenible. James \& James (Science Publishers). Ed. Gustavo Gili, SL. $1^{a} \underline{a}$ Edición, $4^{\underline{a}}$ tirada.

Vitruvio M.V. (2000). Los diez libros de Arquitectura. Obras maestras. Iberia. 